\title{
Trabajo de campo en la virtualidad. Un dispositivo grupal posible
}

\author{
Field work in virtuality. A posible group technique
}

María Cecilia Nogueira

https://orcid.org/0000-0002-9487-4520

mcecilianogueira123@gmail.com

Facultad de Trabajo Social |

Universidad Nacional de La Plata | Argentina

\section{Galia Savino}

https://orcid.org/0000-0003-1902-2819

gaalsavino@hotmail.com

Facultad de Trabajo Social I

Universidad Nacional de La Plata | Argentina

\author{
Carina Scharagrodsky \\ https://orcid. org/0000-0002-9487-4520 \\ carinosky@hotmail.com \\ Instituto de Estudios en Trabajo Social y Sociedad \\ Facultad de Trabajo Social | UNLP | Argentina
}

\author{
Florencia Dutria \\ https://orcid.org/0000-0002-3301-5220 \\ flordutria96@gmail.com \\ Facultad de Trabajo Social I \\ Universidad Nacional de La Plata | Argentina
}

\section{RESUMEN}

Este trabajo se enmarca en el proyecto de investigación tetra anual "Representaciones sociales sobre "salud" $y$ "trabajo" y sus transformaciones en estudiantes universitaries de la carrera de Trabajo Social (UNLP). Un estudio de cohorte de cursantes de 4to y 5to año 2020-2021 respectivamente". Fue acreditado y financiado en la convocatoria 2018 (UNLP). Su Unidad Ejecutora es el Instituto de Estudios en Trabajo Social y Sociedad (IETSyS) de la Facultad de Trabajo Social. Es el primer proyecto de investigación de la cátedra Salud Colectiva integrado por todas sus docentes, una adscripta, una estudiante, una docente de la FaHCE y una colaboradora externa.

Nos proponemos dar cuenta del desafío metodológico enfrentado en contexto de pandemia (COVID 19) para dar continuidad a la investigación durante 2020, tomando especial consideración en la técnica de recolección de información grupal implementada en el marco de la virtualización de las clases. Nuestro objetivo consiste en describir y analizar de manera preliminar la actividad grupal desarrollada durante el segundo cuatrimestre de este año. Intentaremos compartir cómo planificamos la tarea, qué herramientas se utilizaron, cómo realizamos la convocatoria, cuáles fueron los momentos de la actividad y cómo se llevó a cabo para finalmente esbozar algunos resultados preliminares.

PALABRAS CLAVE

Representaciones Sociales, Salud,

Dispositivo grupal, Trabajo Social. 


\section{KEY WORDS}

Social Representation, Health,

Grouptechnique,

Social Work.

\section{ABSTRACT}

Thisworkispart of theannual tetra researchproject T103 "Social representationsabout" health "and" work "and theirtransformations in universitystudents of the Social Workcareer (UNLP). A cohortstudy of 4th and 5th yearstudents 2020-2021 respectively ". Itwasaccredited and funded in 2018 (UNLP). ItsExecutingUnitisthelnstitute of Studies in Social Work and Society (IETSyS) of theFaculty of Social Work. Itisthefirstresearchproject of theCollectiveHealthchairmade up of allitsteachers, a student, a FaHCEteacher and anexternalcollaborator.

Wepropose to accountforthemethodologicalchallengefaced in thecontext of a pandemic (COVID 19) to continuetheresearchduring 2020, takingspecialconsideration in thegroupinformationcollectiontechniqueimplementedwithintheframework of thevirtualization of theclasses. Ourobjectiveis to describe and analyze in a preliminarywaythegroupactivitydevelopedduringthesecondsemester of thisyear. Wewill try to share howweplannedthetask, whattoolswereused, howwemadethecall, whichwerethemoments of theactivity and howitwascarriedout to finallyoutlinesomepreliminaryresults. 


\section{INTRODUCCIÓN}

Presentaremos el proyecto contemplando: el objetivo, el marco teórico con una mención de las categorías centrales y la metodología. Nos detendremos en una de las técnicas administradas que consistió en un dispositivo grupal con momentos asincrónicos y sincrónicos.

\section{Objetivo:}

El proyecto tiene como objetivo analizar las articulaciones y transformaciones de las representaciones sociales (RS) sobre categorías tales como Salud y Trabajo construidas por les estudiantes de la Licenciatura en Trabajo Social. Para ello nos proponemos identificar dichas RS en dos momentos de sus trayectorias académicas de grado: al promediar su formación universitaria en la UNLP, previo al ingreso a la cursada de la asignatura Salud Colectiva que se encuentra ubicada en cuarto año de dicha carrera y al finalizar la cursada de la carrera en quinto año (Plan de Estudios 2015). Identificar prácticas educativas favorecedoras y/u obstaculizadoras de las mencionadas transformaciones a los fines de incidir en los procesos de profesionalización de estudiantes de Trabajo Social en el campo de la Salud.

\section{Marco teórico:}

En esta propuesta confluyen dos líneas de investigación: 1) La formación universitaria a través de los procesos de profesionalización y 2) El estudio de las Representaciones Sociales en el campo de la Salud desde la Medicina Social Latinoamericana. 
En esta propuesta confluyen dos líneas de investigación: 1) La formación universitaria a través de los procesos de profesionalización y 2) El estudio de las Representaciones Sociales en el campo de la Salud desde la Medicina Social Latinoamericana.

El marco teórico corresponde a la Psicología Educacional, enfoque vygotskiano (Erausquin (2009, 2014)); la Teoría de las RS de Moscovici y Jodelet $(1986,2008)$ y la Salud Colectiva latinoamericana con su prolífica producción. Las categorías teóricas centrales son los procesos de Profesionalización (Fernández Pérez, 2001; Dubar, 2002), las Representaciones Sociales en sus diversos enfoques: procesual de Moscovici $(1961,1973,1979,1981,1989)$ y Jodelet $(1986$, $2000,2008)$, estructural de Abric $(1994,2001)$ y de corte sociológico de Doise $(1986,1990,1992)) y$ Salud integral desde el pensamiento de la Medicina Social/Salud Colectiva (Laurell (1982), Berlinguer (1994, 2002,2007), López Arellano (2005), Breihl (2010, 2011), Granda (2004), Menéndez (1992, 2009)).

\section{Metodología:}

El enfoque metodológico es cualitativo. Se trata de un estudio prospectivo de la cohorte 2020. La propuesta formulada a fines de 2018 contemplaba las siguientes técnicas de recolección de información: encuestas, entrevista a informante clave, grupo focal y recopilación de documentos. Siguiendo el cronograma previsto, las mismas se comenzarían a administrar durante el año 2020. Pero la pandemia de COVID 19 sorprendió al mundo. En Argentina, el Aislamiento Social Preventivo y Obligatorio fue establecido el 19 de marzo de 2020. Dicha medida impactó en la vida cotidiana y particularmente en la vida universitaria cuyo ciclo lectivo estaba por iniciar e implicó la virtualización no elegida de las clases. En este marco, realizamos una revisión metodológica para implementar la recolección de información en el contexto de las cursadas virtuales a través de formularios Google, videollamadas y actividades grupales por plataformas de manera sincrónica.

\section{Construyendo una técnica grupal en contexto de pandemia}

El desafío metodológico que enfrentó el equipo de investigación consistió en continuar con el proyecto en contexto de pandemia. Para ello tuvimos que construir alternativas metodológicamente válidas que nos permitieran "recuperar" aquello inexorablemente perdido (las construcciones colectivas producto de los intercambios presenciales en el aula). De allí surgió un dispositivo grupal que combina tres momentos y que presentaremos a continuación: 
El desafío metodológico que enfrentó el equipo de investigación consistió en continuar con el proyecto en contexto de pandemia. Para ello tuvimos que construir alternativas metodológicamente válidas que nos permitieran "recuperar" aquello inexorablemente perdido (las construcciones colectivas producto de los intercambios presenciales en el aula).

La primera instancia propone la creación de una "enfermedad", respondiendo a los siguientes criterios correspondientes a cinco columnas de un Padlet:

- NOMBRE (¿Cómo llamaría a esta enfermedad?)

- CARACTERÍSTICAS (¿En qué consiste? ¿Cómo se expresa?, etc.)

- CAUSAS (¿Qué la origina?)

- ABORDAJE (Imaginar intervenciones posibles)

- CONTENIDO AUDIOVISUAL (seleccionar una imagen o fotografía que guarde relación con dicha enfermedad o la forma en que se expresa).

El segundo momento constaría de un encuentro sincrónico en la plataforma Zoom, en grupos reducidos de 5 o 6 personas que tenía como objetivo una puesta en común general en la que cada participante pudiera exponer el proceso de creación de la enfermedad presentada en el Padlet, conocer el proceso de otres y permitir el intercambio.

En tercera instancia, la consigna previa al ingreso al dispositivo grupal consistió en revisar la plataforma Padlet producida por cada grupo en las instancias anteriores para responder a la siguiente pregunta: "¿Qué dejarías, que sacarías y qué agregarías a tu propuesta inicial?"

\section{Sobre el desarrollo de la técnica}

\section{a) Las herramientas utilizadas:}

Se desarrolló un instructivo para la resolución del Padlet que se difundió entre les participantes. Se brindó información precisa acerca de cómo insertar los comentarios y el tiempo aproximado que podía insumirles la redacción.

\section{b) La convocatoria:}

Trabajamos en dos líneas: por un lado, los recursos formales con los que contábamos para comunicarnos con les estudiantes; vía mail y aulas web principalmente. A través de esta vía difundimos el ins- 
tructivo e informamos los objetivos de la actividad y los pasos a seguir para participar, entre otros puntos que conllevan una extensión considerable.

Por otro lado, utilizamos recursos informales como la cercanía de parte del equipo con algunes estudiantes, las redes sociales (sobretodo WhatsApp e Instagram), que nos permitieron establecer un diálogo más fluido, llevar el recordatorio de la fecha y la hora a les participantes, confirmar la asistencia a la misma y despejar dudas. De la misma manera creemos que la cercanía entre participantes hizo una especie de fuerza de empuje que alentó a la participación entre elles.

\section{c) Modalidades de registro actividad}

En todos los grupos se designó a un observador no participante y a un observador participante que se abocaron a registrar el transcurso de la técnica. Estas notas dieron cuenta al equipo de los modos (fortalezas y obstáculos) en el desarrollo de los grupos. Los encuentros sincrónicos fueron grabados.

Las actividades del equipo, incluyendo la planificación, fueron registradas a través de crónicas, del intercambio de mensajes y en los espacios sincrónicos generados por las participantes del equipo.

Cuadro 1: Elaboración propia

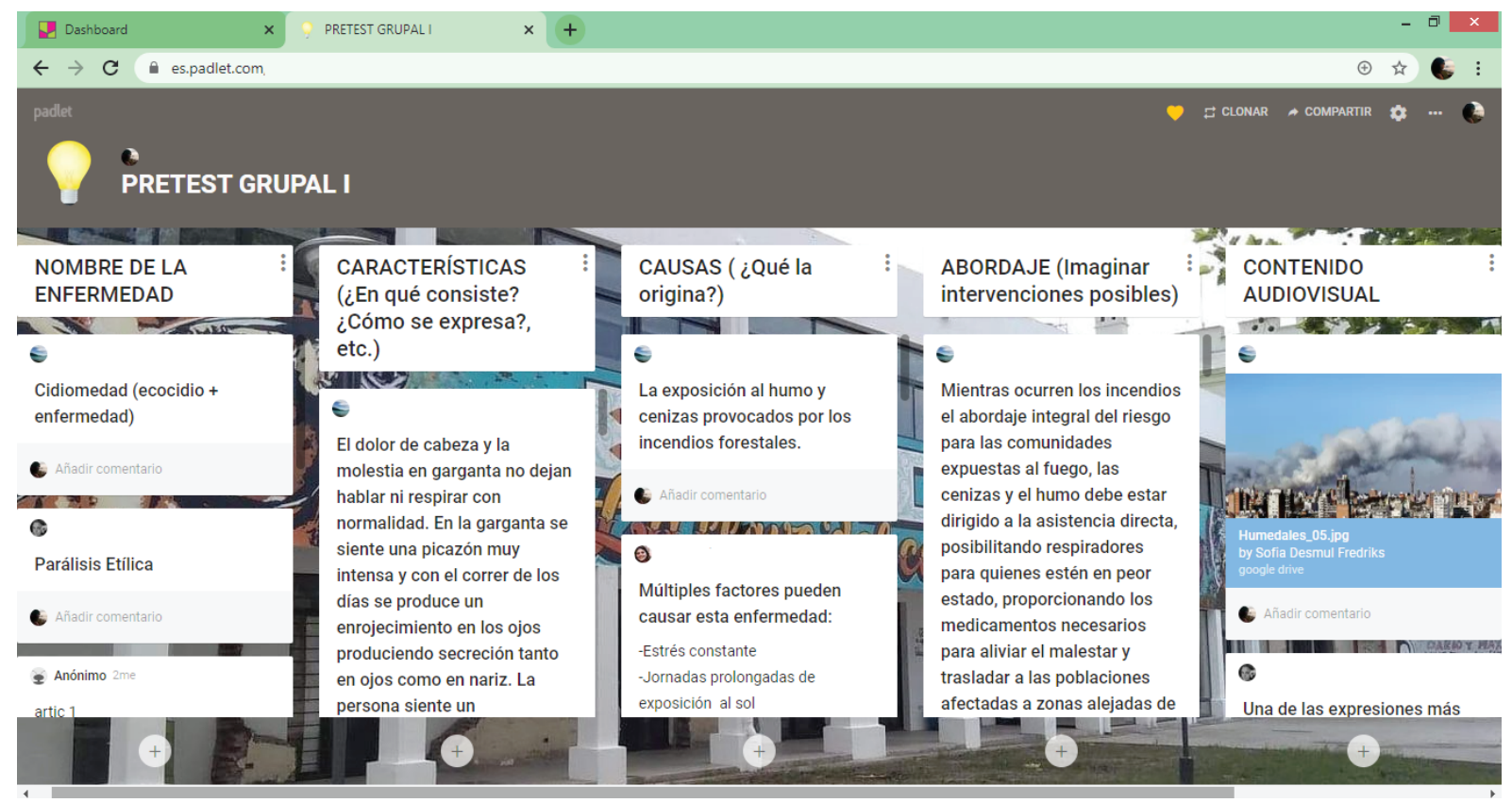

\section{d) Breve síntesis de las crónicas}

Primer encuentro grupal (22/09 entre las 10:00 y las 10:40 hs.).

Las enfermedades propuestas en el Padlet fueron: 
- Cidiomedad (ecocidio + enfermedad) (S)

- Parálisis etílica (M)

- Dermatrofia (A)

- Tabacidad (G)

- Biancionolitis

- Hepatoalergin (A)

Tras breves comentarios de intercambio entre la coordinadora y les participantes, se inicia un primer momento de actividad grupal radial a partir de preguntas como: ¿Qué les pareció la actividad? ¿Cómo la pensaron?

Tras breves comentarios de intercambio entre la coordinadora y les participantes, se inicia un primer momento de actividad grupal radial a partir de preguntas como: ¿Qué les pareció la actividad? ¿Cómo la pensaron?

Les estudiantes comienzan a comentar sus producciones destacando lo difícil que les resultó inventar o proponer algo nuevo: $\mathrm{M}$ ) expresa -"resulta complicado esto de estar inventando o proponiendo algo que ya existe.- (G) dice que le pasó lo mismo: -"Me costó pensar qué es la salud y qué es la enfermedad"..."Estuvo bueno, interesante". (A):- "yo no lo armé sola. Obligué a mi familia a ayudarme a pensar en la enfermedad". M: yo me re divertí... igualmente pedí ayuda"-

A partir de allí comenzaron a identificar las enfermedades propuestas: (S) refirió: -"Me metí con algo que me interesa (cidiomedad), la cuestión ecológica: los incendios en los humedales (...). Pensé en una perspectiva de Salud, desde la salud colectiva porque el lugar en el que vivimos es fundamental. (...) Las condiciones de vida, la salud, el aire. Rosario tiene el peor aire del mundo. Eso no es calidad de vida. Y en La Plata el estrés, lo que comemos...vi que A. puso algo sobre alimentos..."-

(G): -"Para mí el problema (tabacidad) surgió así, de la nada, para darle una vuelta más al tabaco con algo nuevo y también la pandemia. La utilización del barbijo también, salió de ahí (refiriéndose a la imagen de la máscara que había colgado en el Padlet). Es difícil pensar en un abordaje para algo que estaba inventando. No se sabe muy bien la causa. Un abordaje médico, pero después me puse a pensar en concientizar-."

(A): Acerca de la dermatrofia, refirió - "Tiene que ver con un miedo mío a las manchas en la piel. El abordaje lo crucé con el TP3 sobre determinantes sociales y pensé en la cantidad de horas que las personas están expuestas al sol cuando se dedican a la construcción, bajos recursos, mala alimentación... estuve repensando la intervención construida para un sector específico y pensar a partir de los contextos. (...) M quien 
se había referido a la parálisis etílica, continúa con esta idea: -"...pensaba en esto de tener al sol incorporado... Me pasó que fui a Guernica, a las tomas y volví insolada, con mucho dolor de cabeza. El sol hace mucho daño. Después de estar encerrada seis meses (por la pandemia) tuve dolor de cabeza y fiebre por haber estado 506 horas al sol. Enfermedades como las que propuso Ana."

Segundo encuentro grupal (11/11 entre las 16:00 y las 16:40 hs.).

Tras breves comentarios de intercambio entre la coordinadora y les participantes, se inicia el tercer momento de actividad grupal a partir de la siguiente pregunta: ¿Pudieron mirar sus pre producciones? ¿Qué dejarían? ¿Qué cambiarían?

(G) dice que: "al releer el Padlet había contenidos muy básicos. No sé si cambiaría el nombre o las características de la enfermedad, pero si el abordaje(...) Mi sugerencia fue de un abordaje individual: no exponerse al humo del tabaco... Ahora lo pensaría desde un abordaje colectivo (...) Lo había pensado como una cuestión estrictamente médica enmarcada en el Modelo Médico Hegemónico: la causa, el síntoma y el tratamiento (...)".

C (Coord.): "-En el intercambio grupal se generó un ida y vuelta y algo apareció..."

(G):-"Es distinto a lo que podríamos pensar ahora con los parciales entregados y los contenidos trabajados."

(S):-"Ayer respondí el postest y me pasó lo mismo que me pasó acá cuando volví al Padlet. No cambiaría el nombre de la enfermedad, pero si las causas y los abordajes. Había pensado en abordajes propiamente médicos que resuelvan al momento lo que está sucediendo. Hoy puedo darle una vuelta(...) Es increíble, pero una no sabe cómo funciona el sistema de salud en su país. Hoy lo sé y me parece importante. Pensé en cuestiones específicamente médicas, del sentido común: una enfermedad: una patología que se resolviera con un tratamiento médico. (...) Fue un antes y después para mí. Con respecto a la intervención hoy pensaría en la promoción de la salud. Cuando leí que toda política social es una política de salud pude ver la integralidad. Disfruté de la materia y aprendí un montón"

(M): -"En el grupo hablamos de algunas cuestiones. En esta carrera ya tenemos ciertas puntas: las condiciones que afectan nuestra vida cotidiana, ver la enfermedad, la salud y el cuidado como un proceso, pero no teníamos eso aceitado más allá del cuerpo(...)." 
(A): -"El abordaje para mí siempre es más desde lo macro que desde lo micro. Estábamos más centrados en las categorías biológicas y nos fuimos dando cuenta de la integralidad que tiene el proceso. "Toda política social es una política de salud". La enfermedad siempre estaba referida a lo biológico, a lo físico (...)"

(C): Hace alusión al refuerzo familiar solicitado como colaboración para completar el Padlet inicial y les participantes acuerdan con M: - "Hoy capaz frente al Padlet inicial no tendría que pedir ayuda. Es complejo. La violencia de género como problema para la salud. No lo podía ver de esa manera. Ahora tengo otro panorama, otros límites más amplios para pensar en estos términos-"

(S): - "Todo esto me lo llevo para mi formación? Al principio decía: "no me gusta la salud". Hoy digo: "capaz haga algo...". Me acercó. Pensar que "Toda política social es una política de salud" me rompió la cabeza. El MMH para mí es como el patriarcado. El Padlet fue una trampa del MMH: la consigna era ponerle un nombre a la enfermedad y se nos fue para el lado biológico. (...)“"

\section{e) Análisis preliminares:}

A pesar de las dificultades del desarrollo de las actividades en contextos de pandemia, la actividad pudo completarse de manera exitosa.

Consideramos que el hecho de que les estudiantes se conocieran previamente (recordemos que es una materia de cuarto año) contribuyó a que se sintieran cómodos y habilitados para participar, expresarse libremente sin ser juzgados.

A partir del análisis en el manejo del tiempo del primer grupo, el corte fue un tanto abrupto a los 40 minutos de iniciado el mismo, en la segunda instancia, comenzamos el cierre unos minutos antes y pudimos finalizar el espacio de un modo más prolijo.

Con respecto a las voces de les estudiantes, sus miradas y sus perspectivas en relación a los ejes trabajados en el proyecto, pueden identificarse dos momentos muy diferenciados en relación a sus consideraciones acerca de la salud, enfermedad, sus causas, las estrategias de abordaje y la relación con el contexto económico, social, político y cultural.

Con respecto a las voces de les estudiantes, sus miradas y sus perspectivas en relación a los ejes trabajados en el proyecto, pueden identificarse dos momentos muy diferenciados en relación a sus consideraciones acerca de la salud, enfermedad, sus causas, las es- 
trategias de abordaje y la relación con el contexto económico, social, político y cultural.

En el primer encuentro se destacó el atravesamiento biomédico en relación a algunas de las enfermedades propuestas y a las intervenciones donde asoma tímidamente la dimensión social y la responsabilidad del Estado a través de las políticas públicas. Aparecieron los determinantes sociales tras la alusión al momento de la cursada en la que se solicitó un TP obligatorio sobre este tema sin profundizar acerca de la dimensión teórica de la categoría. Las temáticas van madurando durante el intercambio entre les estudiantes, instalando cuestiones vinculadas a la salud colectiva y atravesadas por las trayectorias singulares (militancia) de les participantes. Aparece el coronavirus, sin embargo, NO domina la escena de temas propuestos.

En el segundo encuentro identifican como "básicas o simples" a sus primeras propuestas en el Padlet. Marcan con claridad las transformaciones: hay un antes y un después en sus producciones especialmente en lo que hace a los abordajes y las causas y fundamentan teóricamente esas modificaciones.

Resultó llamativo que articularon todas las técnicas utilizadas tanto por el proyecto de investigación (pretest/postest) como la resolución de las consignas de los dos parciales. Atribuyen a la disciplina del TS la visión social, pero a la asignatura la redimensión de los procesos de s/e/a en contraposición con el MMH que los atravesó con claridad para interpretar el primer momento de la actividad. Aparece el tema del coronavirus, pero no como un tema central. Recuperan y destacan como valiosos los contenidos de la materia.

El presente documento constituye un análisis preliminar en relación a la experiencia de recolección de información tal como lo teníamos estipulado en el proyecto, adaptando las técnicas a contextos que no tienen precedentes.

Desarrollar grupos de discusión en un espacio virtual, no consiste en trasladar una técnica de un espacio a otro. Representa un desafío teórico y metodológico cuyas dimensiones son también éticas y políticas. Implicó preguntarnos como equipo cuestiones: ¿Es posible desarrollar tareas de campo en este contexto? ¿La virtualidad le quitará posibilidades a la interacción de les participantes?

Esperamos encontrar las respuestas a estas preguntas a partir de la reflexión que surge de los debates entre el equipo y su práctica con otres. 


\section{BIBLIOGRAFÍA}

Abric, J. (2001). "Metodología de recolección de las representaciones sociales". En J. Abric (Coord.). Prácticas sociales y representaciones. México: Ediciones Coyoacán, p. 53-74.

Charaudeau, P. \& Maingueneau, D. (Dirs.) (2005). “Diccionario de análisis del discurso". Buenos Aires: Nueva Visión.

Dubar, C. (2001). “El trabajo y las identidades profesionales y personales". En: Revista Latinoamericana de Estudios del Trabajo, Año 13 (7). Buenos Aires.

Dussel, I. (2020). "La clase en pantuflas". Conversatorio virtual organizado en el espacio Diálogos sobre pedagogía, Buenos Aires: Instituto Superior de Estudios Pedagógicos. Disponible en: https://www.youtube.com/watch?$v=6 x K v C t B C 3 V s$.

Erausquin C. y D' Árcangelo M. (2018). “Unidades de Análisis para la construcción de conocimientos e intervenciones en escenarios educativos". En Interpelando entramados de experiencias. Cruce de fronteras e implicación psico-educativa entre universidad y escuelas. La Plata, Provincia de Buenos Aires (Argentina). Editorial de la Universidad de La Plata: EDULP.

Iriart, C.; Waitzkin, H.; Breilh, J.; Estrada A. \& Merhy, E. (2002). “Medicina Social Latinoamericana. Aportes y Desafíos" En: Revista Panamericana de Salud Pública. v.12(2) p.128-136.

Jodelet, D. (2000). "Representaciones sociales: contribución a un saber sociocultural sin fronteras". En D. Jodelet y A. Guerrero, Develando la cultura. Estudios en representaciones sociales. México: Facultad de Psicología-UNAM, p. 7-30.

Menéndez. E. (2009). “De sujetos, saberes y estructuras. Introducción al enfoque relacional en el estudio de la salud colectiva". Buenos Aires: Lugar editorial.

Moscovici, S. (1989). "Des representations collectives aux representations sociales: elementes pour une historie". En D. Jodelet (Ed.), Les representations sociales. Paris: PUF. 